\title{
On the well-posedness of the incompressible porous media equation in Triebel-Lizorkin spaces
}

Wenxin $\mathrm{Yu}^{1 *}$ and Yigang $\mathrm{He}^{2}$

\section{"Correspondence:}

slowbird@sohu.com

${ }^{1}$ College of Electrical and

Information Engineering, Hunan

University, Changsha, 410000, P.R. China

Full list of author information is

available at the end of the article

\begin{abstract}
In this paper, we prove the local well-posedness for the incompressible porous media equation in Triebel-Lizorkin spaces and obtain blow-up criterion of smooth solutions. The main tools we use are the Fourier localization technique and Bony's paraproduct decomposition.

MSC: $76505 ; 76 \mathrm{D03}$

Keywords: well-posedness; incompressible porous media equation; blow-up criterion; Fourier localization; Bony's paraproduct decomposition; Triebel-Lizorkin space
\end{abstract}

\section{Introduction}

In this paper, we are concerned with the incompressible porous media equation (IPM) in $\mathbb{R}^{d}(d=2$ or 3$)$ :

$$
\left\{\begin{array}{lr}
\partial_{t} \theta+u \cdot \nabla \theta=0, & \theta(x, 0)=\theta_{0}, \\
u=-k(\nabla p+g \gamma \theta), & \operatorname{div} u=0,
\end{array}\right.
$$

where $x \in \mathbb{R}^{d}, t>0, \theta$ is the liquid temperature, $u$ is the liquid discharge, $p$ is the scalar pressure, $k$ is the matrix of position-independent medium permeabilities in the different directions, respectively, divided by the viscosity, $g$ is the acceleration due to gravity, and $\gamma \in \mathbb{R}^{d}$ is the last canonical vector $e_{d}$. For simplicity, we only consider $k=g=1$.

By rewriting Darcy's law we obtain the expression of velocity $u$ only in terms of temperature $\theta[1,2]$. In the 2D case, thanks to the incompressibility, taking the curl operator first and the $\nabla^{\perp}:=\left(-\partial_{x_{2}}, \partial_{x_{1}}\right)$ operator second on both sides of Darcy' law, we have

$$
-\Delta u=\nabla^{\perp}\left(\partial_{x_{1}} \theta\right)=\left(-\partial_{x_{1}} \partial_{x_{2}} \theta, \partial_{x_{1}}^{2} \theta\right)
$$

thus the velocity $u$ can be recovered as

$$
u(x, t)=-\frac{1}{2 \pi} \int_{\mathbb{R}^{2}} \ln |x-y|\left(-\frac{\partial^{2} \theta}{\partial y_{2} \partial y_{1}}(y, t), \frac{\partial^{2} \theta}{\partial^{2} y_{1}}(y, t)\right) d y, \quad x \in \mathbb{R}^{2} .
$$

@2014 Yu and He; licensee Springer. This is an Open Access article distributed under the terms of the Creative Commons Attribution License (http://creativecommons.org/licenses/by/2.0), which permits unrestricted use, distribution, and reproduction in any medium, provided the original work is properly cited. 
Through integration by parts we finally get

$$
u(x, t)=-\frac{1}{2}(0, \theta(x, t))+\frac{1}{2 \pi} P V \int_{\mathbb{R}^{2}} H(x-y) \theta(y, t) d y, \quad x \in \mathbb{R}^{2},
$$

where the kernel $H(\cdot)$ is defined by

$$
H(x)=\left(\frac{2 x_{1} x_{2}}{|x|^{4}}, \frac{x_{2}^{2}-x_{1}^{2}}{|x|^{4}}\right) \text {. }
$$

Similarly, in 3D case, applying the curl operator twice to Darcy's law, we get

$$
-\Delta u=\left(-\partial_{1} \partial_{3} \theta,-\partial_{2} \partial_{3} \theta, \partial_{1}^{2} \theta+\partial_{2}^{2} \theta\right),
$$

where $\partial_{i}:=\frac{\partial}{\partial x_{i}}$, thus

$$
u(x, t)=-\frac{2}{3}(0,0, \theta(x, t))+\frac{1}{4 \pi} P V \int_{\mathbb{R}^{3}} K(x-y) \theta(y, t) d y, \quad x \in \mathbb{R}^{3},
$$

where

$$
K(x)=\left(\frac{3 x_{1} x_{3}}{|x|^{5}}, \frac{3 x_{2} x_{3}}{|x|^{5}}, \frac{2 x_{3}^{2}-x_{1}^{2}-x_{2}^{2}}{|x|^{5}}\right) .
$$

We observe that, in general, each coefficient of $u(\cdot, t)$ (with $t$ as parameter) is only the linear combination of the Calderon-Zygmund singular integral (with the definition see the sequel) of $\theta$ and $\theta$ itself. We write the general version as

$$
u:=\mathcal{T}(\theta)=\mathcal{C}(\theta)+\mathcal{S}(\theta)
$$

where $\mathcal{T}=\left(\mathcal{T}_{k}\right), \mathcal{C}=\left(\mathcal{C}_{k}\right), \mathcal{S}=\left(\mathcal{S}_{k}\right), 1 \leq k \leq N$ are all operators mapping scalar functions to vector-valued functions and $\mathcal{C}_{k}$ equals a constant multiplication operator whereas $\mathcal{S}_{k}$ means a Calderón-Zygmund singular integral operator. Especially the corresponding specific forms in 2D or 3D are shown as (1.2) or (1.3).

We observe that the system (IPM) is not more than a transport equation with non-local divergence-free velocity field (the specific relationship between velocity and temperature as (1.4) shows). It shares many similarities with another flow model - the 2D dissipative quasi-geostrophic $(\mathrm{QG})$ equation, which has been intensively studied by many authors [3-8]. From a mathematical point of view, the system (IPM) is somewhat a generalization of the (QG) equation. Very recently, the system (IPM) was introduced and investigated by Córdoba et al. In [2], they treated the (IMP) in 2D case and obtained the local existence and uniqueness in Hölder space $C^{\delta}$ for $0<\delta<1$ by the particle-trajectory method and gave some blow-up criteria of smooth solutions. Recently, they proved non-uniqueness for weak solutions of (IPM) in [9]. For the dissipative system related (IPM), in [1], the authors obtained some results on strong solutions, weak solutions and attractors. For finite energy they obtained global existence and uniqueness in the subcritical and critical cases. In the supercritical case, they obtained local results in $H^{s}, s>(N-\alpha) / 2+1$ and extended to be global under a small condition $\left\|\theta^{0}\right\|_{H^{s}} \leq c v$, for $s>N / 2+1$, where $c$ is a small fixed constant. 
Recently, Chae studied the local well-posedness and blow-up criterion for the incompressible Euler equations [10,11], and quasi-geostrophic equations [12] in Triebel-Lizorkin spaces. As is well known, Triebel-Lizorkin spaces are the unification of several classical function spaces such as Lebegue spaces $L^{p}\left(\mathbb{R}^{d}\right)$, Sobolev spaces $H_{p}^{s}\left(\mathbb{R}^{d}\right)$, Lipschitz spaces $C^{s}\left(\mathbb{R}^{d}\right)$, and so on. In [10], the author first used the Littlewood-Paley operator to localize the Euler equation to the frequency annulus $\left\{|\xi| \sim 2^{j}\right\}$, then obtained an integral representation of the frequency-localized solution on the Lagrangian coordinates by introducing a family of particle-trajectory mappings $\left\{X_{j}(\alpha, t)\right\}$ defined by

$$
\left\{\begin{array}{l}
\frac{\partial}{\partial t} X_{j}(\alpha, t)=\left(S_{j-2} v\right)\left(X_{j}(\alpha, t), t\right) \\
X_{j}(\alpha, 0)=\alpha
\end{array}\right.
$$

where $v$ is a divergence-free velocity field and $S_{j-2}$ is a frequency projection to the ball $\left\{|\xi| \lesssim 2^{j}\right\}$ (see Section 2). He also used the following equivalent relation:

$$
\left\|\left(\sum_{j \in \mathbb{Z}} 2^{j s q}\left|\Delta_{j} v\left(X_{j}(\alpha, t)\right)\right|^{q}\right)^{\frac{1}{q}}\right\|_{L^{p}(\cdot d \alpha)} \cong\left\|\left(\sum_{j \in \mathbb{Z}} 2^{j s q}\left|\Delta_{j} v(x)\right|^{q}\right)^{\frac{1}{q}}\right\|_{\left.L^{p} \cdot d x\right)}=\|v\|_{\dot{F}_{p, q}^{s}}
$$

to estimate the frequency-localized solutions of the Euler equations or quasi-geostrophic equations in Triebel-Lizorkin spaces. However, it seems difficult to give a strict proof for the above equivalent relation (1.6) and its related counterpart due to the lack of a uniform change of the coordinates independent of $j$. To avoid this trouble, Chen et al. [13] introduced a particle trajectory mapping $X(\alpha, t)$ independent of $j$ defined by

$$
\left\{\begin{array}{l}
\frac{\partial}{\partial t} X(\alpha, t)=v(X(\alpha, t), t) \\
X(\alpha, 0)=\alpha
\end{array}\right.
$$

and then established a new commutator estimate to obtain the local well-posedness of the ideal MHD equations in the Triebel-Lizorkin spaces.

In this paper, we will adapt the method of Chen et al. [13] to establish the local wellposedness for the incompressible porous media equation (1.1) and to obtain a blow-up criterion of smooth solutions in the framework of Triebel-Lizorkin spaces.

Now we state our result as follows.

Theorem 1.1 (i) Local-in-time existence. Let $s>\frac{d}{p}+1,1<p, q<\infty$. Assume that $\theta_{0} \in$ $F_{p, q}^{s}\left(\mathbb{R}^{d}\right)$, then there exists $T=T\left(\left\|\theta_{0}\right\|_{F_{p, q}^{s}}\right)$ such that (1.1) has a unique solution $\theta \in$ $C\left([0, T] ; F_{p, q}^{s}\left(\mathbb{R}^{d}\right)\right) \cap \operatorname{Lip}\left([0, T] ; F_{p, q}^{s-1}\left(\mathbb{R}^{d}\right)\right)$.

(ii) Blow-up criterion. The local-in-time solution $\theta \in C\left([0, T] ; F_{p, q}^{s}\right)$ constructed in (i) blows up at $T^{*}>T$ in $F_{p, q}^{s}$, i.e.

$$
\underset{t \nearrow T^{*}}{\limsup }\|\theta(t)\|_{F_{p, q}^{s}}=+\infty, \quad T^{*}<\infty,
$$

if and only if

$$
\int_{0}^{T^{*}}\|\theta(t)\|_{\dot{F}_{\infty, \infty}^{1}} d t=+\infty
$$




\section{Preliminaries}

Let $\mathcal{B}=\left\{\xi \in \mathbb{R}^{d},|\xi| \leq \frac{4}{3}\right\}$ and $\mathcal{C}=\left\{\xi \in \mathbb{R}^{d}, \frac{3}{4} \leq|\xi| \leq \frac{8}{3}\right\}$. Choose two nonnegative smooth radial functions $\chi, \varphi$ supported, respectively, in $\mathcal{B}$ and $\mathcal{C}$ such that

$$
\chi(\xi)+\sum_{j \geq 0} \varphi\left(2^{-j} \xi\right)=1, \quad \xi \in \mathbb{R}^{d}, \quad \sum_{j \in \mathbb{Z}} \varphi\left(2^{-j} \xi\right)=1, \quad \xi \in \mathbb{R}^{d} \backslash\{0\} .
$$

We denote $\varphi_{j}(\xi)=\varphi\left(2^{-j} \xi\right), h=\mathcal{F}^{-1} \varphi$ and $\tilde{h}=\mathcal{F}^{-1} \chi$. Then the dyadic blocks $\Delta_{j}$ and $S_{j}$ can be defined as follows:

$$
\begin{aligned}
& \Delta_{j} f=\varphi\left(2^{-j} D\right) f=2^{j d} \int_{\mathbb{R}^{d}} h\left(2^{j} y\right) f(x-y) d y, \\
& S_{j} f=\sum_{k \leq j-1} \Delta_{k} f=\chi\left(2^{-j} D\right) f=2^{j d} \int_{\mathbb{R}^{d}} \tilde{h}\left(2^{j} y\right) f(x-y) d y .
\end{aligned}
$$

Formally, $\Delta_{j}=S_{j+1}-S_{j}$ is a frequency projection to the annulus $\left\{|\xi| \sim 2^{j}\right\}$, and $S_{j}$ is a frequency projection to the ball $\left\{|\xi| \lesssim 2^{j}\right\}$. One easily verifies that with our choice of $\varphi$

$$
\Delta_{j} \Delta_{k} f \equiv 0 \quad \text { if }|j-k| \geq 2 \quad \text { and } \quad \Delta_{j}\left(S_{k-1} f \Delta_{k} f\right) \equiv 0 \quad \text { if }|j-k| \geq 5 .
$$

With the introduction of $\Delta_{j}$ and $S_{j}$, let us recall the definition of the Triebel-Lizorkin space. Let $s \in \mathbb{R},(p, q) \in[1, \infty) \times[1, \infty]$, the homogeneous Triebel-Lizorkin space $\dot{F}_{p, q}^{s}$ is defined by

$$
\dot{F}_{p, q}^{s}=\left\{f \in \mathcal{Z}^{\prime}\left(\mathbb{R}^{d}\right) ;\|f\|_{\dot{F}_{p, q}^{s}}<\infty\right\},
$$

where

$$
\|f\|_{\dot{F}_{p, q}^{s}}= \begin{cases}\left\|\left(\sum_{j \in \mathbb{Z}} 2^{j s q}\left|\Delta_{j} f\right|^{q}\right)^{\frac{1}{q}}\right\|_{L^{p}}, & \text { for } 1 \leq q<\infty, \\ \left\|\sup _{j \in \mathbb{Z}}\left(2^{j s}\left|\Delta_{j} f\right|\right)\right\|_{L^{p}}, & \text { for } q=\infty,\end{cases}
$$

and $\mathcal{Z}^{\prime}\left(\mathbb{R}^{d}\right)$ denotes the dual space of $\mathcal{Z}\left(\mathbb{R}^{d}\right)=\left\{f \in \mathcal{S}\left(\mathbb{R}^{d}\right) ; \partial^{\alpha} \hat{f}(0)=0 ; \forall \alpha \in \mathbb{N}^{d}\right.$ multi-index $\}$ and can be identified by the quotient space of $\mathcal{S}^{\prime} / \mathcal{P}$ with the polynomials space $\mathcal{P}$.

For $s>0$ and $(p, q) \in[1, \infty) \times[1, \infty]$, we define the inhomogeneous Triebel-Lizorkin space $F_{p, q}^{s}$ as follows:

$$
F_{p, q}^{s}=\left\{f \in \mathcal{S}^{\prime}\left(\mathbb{R}^{d}\right) ;\|f\|_{F_{p, q}^{s}<\infty}\right\},
$$

where

$$
\|f\|_{F_{p, q}^{s}}=\|f\|_{L^{p}}+\|f\|_{\dot{F}_{p, q}^{s}} .
$$

We refer to [14] for more details.

Lemma 2.1 (Bernstein's inequality) [15] Let $k \in \mathbb{N}$. There exists a constant $C$ independent off and $j$ such that, for all $1 \leq p \leq q \leq \infty$, the following inequalities hold:

$$
\begin{aligned}
& \operatorname{supp} \hat{f} \subset\left\{|\xi| \lesssim 2^{j}\right\} \quad \Rightarrow \quad \sup _{|\alpha|=k}\left\|\partial^{\alpha} f\right\|_{L^{q}} \leq C 2^{j k+j d\left(\frac{1}{p}-\frac{1}{q}\right)}\|f\|_{L^{p}}, \\
& \operatorname{supp} \hat{f} \subset\left\{|\xi| \sim 2^{j}\right\} \quad \Rightarrow \quad\|f\|_{L^{p}} \leq C \sup _{|\alpha|=k} 2^{-j k}\left\|\partial^{\alpha} f\right\|_{L^{p}} .
\end{aligned}
$$


Lemma 2.2 [14] For any $k \in \mathbb{N}$, there exists a constant $C_{k}$ such that the following inequality holds:

$$
C_{k}^{-1}\left\|\nabla^{k} f\right\|_{\dot{F}_{p, q}^{s}} \leq\|f\|_{\dot{F}_{p, q}^{s+k}} \leq C_{k}\left\|\nabla^{k} f\right\|_{\dot{F}_{p, q}^{s}}
$$

Proposition $2.1[10]$ Let $s>0,(p, q) \in(1, \infty) \times(1, \infty]$, or $p=q=\infty$, then there exists a constant $C$ such that

$$
\begin{aligned}
& \|f g\|_{\dot{F}_{p, q}^{s}} \leq C\left(\|f\|_{L^{\infty}}\|g\|_{\dot{F}_{p, q}^{s}}+\|g\|_{L^{\infty}}\|f\|_{\dot{F}_{p, q}^{s}}\right), \\
& \|f g\|_{F_{p, q}^{s}} \leq C\left(\|f\|_{L^{\infty}}\|g\|_{F_{p, q}^{s}}+\|g\|_{L^{\infty}}\|f\|_{F_{p, q}^{s}}\right) .
\end{aligned}
$$

Proposition $2.2[10]$ Let $s>d / 2$ with $p, q \in[1, \infty]$. Suppose $f \in F_{p, q}^{s}$, then there exists a constant $C$ such that the following inequality holds:

$$
\|f\|_{L^{\infty}} \leq C\left(1+\|f\|_{\dot{F}_{\infty, \infty}^{s}}\left(\log ^{+}\|f\|_{F_{p, q}^{s}}+1\right)\right) .
$$

Proposition $2.3[13]$ Let $(p, q) \in(1, \infty) \times(1, \infty]$, or $p=q=\infty$, and $f$ be a solenoidal vector field. Then for $s>0$

$$
\|\| 2^{k s}\left(\left[f, \Delta_{k}\right] \cdot \nabla g\right)\left\|_{\ell^{q}(\mathbb{Z})}\right\|_{L^{p}} \lesssim\left(\|\nabla f\|_{L^{\infty}}\|g\|_{\dot{F}_{p, q}^{s}}+\|\nabla g\|_{L^{\infty}\|f\|_{\dot{F}_{p, q}^{s}}}\right)
$$

orfor $s>-1$

$$
\|\| 2^{k s}\left(\left[f, \Delta_{k}\right] \cdot \nabla g\right)\left\|_{\ell q(\mathbb{Z})}\right\|_{p} \lesssim\left(\|\nabla f\|_{L^{\infty}}\|g\|_{\dot{F}_{p, q}^{s}}+\|g\|_{L^{\infty}}\|\nabla f\|_{\dot{F}_{p, q}^{s}}\right) .
$$

The classical Calderón-Zygmund singular integrals are operators of the form

$$
T_{c z} f(x):=P V \int_{\mathbb{R}^{N}} \frac{\Omega\left(y^{\prime}\right)}{|y|^{N}} f(x-y) d y=\lim _{\epsilon \rightarrow 0} \int_{|y|>\epsilon} \frac{\Omega\left(y^{\prime}\right)}{|y|^{N}} f(x-y) d y,
$$

where $\Omega$ is defined on the unit sphere of $\mathbb{R}^{N}, \mathbb{S}^{N-1}$, and is integrable with zero average and where $y^{\prime}:=\frac{y}{|y|} \in \mathcal{S}^{N-1}$. Clearly, the definition is meaningful for Schwartz functions. Moreover if $\Omega \in C^{1}\left(\mathcal{S}^{N-1}\right), T_{c z}$ is $L^{p}$ bounded, $1<p<\infty$.

The general version (1.4) of the relationship between $u$ and $\theta$ is in fact ensured by the following result (see e.g. [16]).

Lemma 2.3 Let $m \in C^{\infty}\left(\mathbb{R}^{N} \backslash\{0\}\right)$ be a homogeneous function of degree 0 , and $T_{m}$ be the corresponding multiplier operator defined by $\left(T_{m} f\right)^{\wedge}=m \hat{f}$, then there exist $a \in \mathbb{C}$ and $\Omega \in$ $C^{\infty}\left(\mathbb{S}^{N-1}\right)$ with zero average such that for any Schwartz function $f$,

$$
T_{m} f=a f+P V \frac{\Omega\left(x^{\prime}\right)}{|x|^{N}} * f .
$$

Remark 2.1 Since $-\Delta v=\left(\partial_{1} \partial_{N} \theta, \ldots,-\partial_{N-1} \partial_{N} \theta, \partial_{1}^{2} \theta+\cdots+\partial_{N-1}^{2} \theta\right)$, the Fourier multiplier of the operator $\mathcal{T}$ is rather clear. In fact, each component of its multiplier is the linear combination of the term like $\frac{\xi_{i} \xi_{j}}{|\xi|^{2}}, i, j \in\{1,2, \ldots, N\}$, which of course belongs to $C^{\infty}\left(\mathbb{R}^{N} \backslash\{0\}\right)$ and is homogeneous of degree 0 . 


\section{Proof of Theorem 1.1}

We divide the proof of Theorem 1.1 into several steps.

Step 1. A priori estimates.

Taking the operation $\Delta_{k}$ on both sides of the first equation of (1.1), we have

$$
\partial_{t} \Delta_{k} \theta+u \cdot \nabla \Delta_{k} \theta=u \cdot \nabla \Delta_{k} \theta-\Delta_{k}(u \nabla \theta) \triangleq\left[u, \Delta_{k}\right] \cdot \nabla \theta .
$$

Let $X(\alpha, t)$ be the solution of the following ordinary differential equations:

$$
\left\{\begin{array}{l}
\partial_{t} X(\alpha, t)=u(X(\alpha, t), t) \\
X(\alpha, 0)=\alpha
\end{array}\right.
$$

Then it follows from (3.1) that

$$
\frac{d}{d t} \Delta_{k} \theta(X(\alpha, t), t)=\left[u, \Delta_{k}\right] \cdot \nabla \theta(X(\alpha, t), t)
$$

which implies that

$$
\left|\Delta_{k} \theta(X(\alpha, t), t)\right| \leq\left|\Delta_{k} \theta_{0}(\alpha)\right|+\int_{0}^{t}\left|\left(\left[u, \Delta_{k}\right] \cdot \nabla \theta\right)(X(\alpha, \tau), \tau)\right| d \tau .
$$

Multiplying $2^{k s}$, taking the $\ell^{q}(\mathbb{Z})$ norm on both sides of (3.4), we get by using the Minkowski inequality

$$
\begin{aligned}
& \left(\sum_{k}\left|2^{k s} \Delta_{k} \theta(X(\alpha, t), t)\right|^{q}\right)^{\frac{1}{q}} \\
& \quad \leq\left(\sum_{k}\left|2^{k s} \Delta_{k} \theta_{0}(\alpha)\right|^{q}\right)^{\frac{1}{q}}+\int_{0}^{t}\left(\sum_{k}\left|2^{k s}\left(\left[u, \Delta_{k}\right] \cdot \nabla \theta\right)(X(\alpha, \tau), \tau)\right|^{q}\right)^{\frac{1}{q}} d \tau .
\end{aligned}
$$

Next, taking the $L^{p}$ norm with respect to $\alpha \in \mathbb{R}^{d}$ on both sides of (3.5), we get by using the Minkowski inequality that

$$
\begin{aligned}
& \left(\int_{\mathbb{R}^{d}}\left|\left(\sum_{k}\left|2^{k s} \Delta_{k} \theta(X(\alpha, t), t)\right|^{q}\right)^{\frac{1}{q}}\right|^{p} d \alpha\right)^{\frac{1}{p}} \\
& \quad \leq\left\|\theta_{0}\right\|_{\dot{F}_{p, q}^{s}}+\int_{0}^{t}\left(\int_{\mathbb{R}^{d}}\left|\left(\sum_{k}\left|2^{k s}\left(\left[u, \Delta_{k}\right] \cdot \nabla \theta\right)(X(\alpha, \tau), \tau)\right|^{q}\right)^{\frac{1}{q}}\right|^{p} d \alpha\right)^{\frac{1}{p}} d \tau .
\end{aligned}
$$

Using the fact that $\alpha \mapsto X(\alpha, t)$ is a volume-preserving diffeomorphism due to $\operatorname{div} u=0$, we get from (3.6) that

$$
\|\theta(t)\|_{\dot{F}_{p, q}^{s}} \leq\left\|\theta_{0}\right\|_{\dot{F}_{p, q}^{s}}+\int_{0}^{t}\|\| 2^{k s}\left(\left[u, \Delta_{k}\right] \cdot \nabla \theta\right)\left\|_{\ell^{q}(k \in \mathbb{Z})}\right\|_{L^{p}} d \tau .
$$

Thanks to Proposition 2.3, the last term on the right side of (3.7) is dominated by

$$
\int_{0}^{t}\left(\|\nabla u\|_{L^{\infty}}\|\theta\|_{\dot{F}_{p, q}^{s}}+\|\nabla \theta\|_{L^{\infty}}\|u\|_{\dot{F}_{p, q}^{s}}\right) d \tau
$$


and thus

$$
\|\theta(t)\|_{\dot{F}_{p, q}^{s}} \leq\left\|\theta_{0}\right\|_{\dot{F}_{p, q}^{s}}+\int_{0}^{t}\left(\|\nabla u\|_{L^{\infty}}+\|\nabla \theta\|_{L^{\infty}}\right)\|\theta\|_{\dot{F}_{p, q}^{s}} d \tau,
$$

where we used (1.4) and the boundedness of the Calderón-Zygmund singular integral operator on $\dot{F}_{p, q}^{s}$.

Now from (1.1) we have immediately

$$
\|\theta(t)\|_{L^{p}}=\left\|\theta_{0}\right\|_{L^{p}}
$$

for all $1 \leq p \leq \infty$, since div $u=0$. Summing up (3.9) and (3.10) yields

$$
\|\theta(t)\|_{F_{p, q}^{s}} \leq\left\|\theta_{0}\right\|_{F_{p, q}^{s}}+\int_{0}^{t}\left(\|\nabla u\|_{L^{\infty}}+\|\nabla \theta\|_{L^{\infty}}\right)\|\theta\|_{F_{p, q}^{s}} d \tau,
$$

which together with the Gronwall inequality gives

$$
\|\theta(t)\|_{F_{p, q}^{s}} \leq\left\|\theta_{0}\right\|_{F_{p, q}^{s}} \exp \left(C \int_{0}^{t}\left(\|\nabla u\|_{L^{\infty}}+\|\nabla \theta\|_{L^{\infty}}\right) d \tau\right) .
$$

Step 2. Approximate solutions and uniform estimates.

We construct the approximate solutions of (1.1). Define the sequence $\left\{\theta^{(n)}, u^{(n)}\right\}_{\mathbb{N}_{0}=\mathbb{N} \cup\{0\}}$ by solving the following systems:

$$
\left\{\begin{array}{l}
\partial_{t} \theta^{(n+1)}+u^{(n)} \cdot \nabla \theta^{(n+1)}=0, \\
u^{(n)}=\mathcal{C}\left(\theta^{(n)}\right)+\mathcal{S}\left(\theta^{(n)}\right), \\
\operatorname{div} u^{(n)}=0, \\
\left.\theta^{(n+1)}\right|_{t=0}=S_{n+2} \theta_{0} .
\end{array}\right.
$$

We set $\left(\theta^{(0)}, u^{(0)}\right)=(0,0)$ and let $X^{(n)}(\alpha, t)$ be the solutions of the following ordinary differential equations:

$$
\left\{\begin{array}{l}
\partial_{t} X^{(n)}(\alpha, t)=u^{(n)}\left(X^{(n)}(\alpha, t), t\right) \\
X^{(n)}(\alpha, 0)=\alpha
\end{array}\right.
$$

for each $n \in \mathbb{N}$. Then, following the same procedure of estimate leading to (3.11), we obtain

$$
\begin{aligned}
& \left\|\theta^{(n+1)}(t)\right\|_{F_{p, q}^{s}} \\
& \quad \leq\left\|S_{n+2} \theta_{0}\right\|_{F_{p, q}^{s}}+\int_{0}^{t}\left(\left\|\nabla u^{(n)}\right\|_{L^{\infty}}\left\|\theta^{(n+1)}\right\|_{F_{p, q}^{s}}+\left\|\nabla \theta^{(n+1)}\right\|_{L^{\infty}}\left\|u^{(n)}\right\|_{F_{p, q}^{s}}\right) d \tau \\
& \quad \leq\left\|\theta_{0}\right\|_{F_{p, q}^{s}}+\int_{0}^{t}\left(\left\|\nabla u^{(n)}\right\|_{F_{p, q}^{s-q}}\left\|\theta^{(n+1)}\right\|_{F_{p, q}^{s}}+\left\|\nabla \theta^{(n+1)}\right\|_{F_{p, q}^{s-q}}\left\|u^{(n)}\right\|_{F_{p, q}^{s}}\right) d \tau \\
& \quad \leq\left\|\theta_{0}\right\|_{F_{p, q}^{s}}+\int_{0}^{t}\left\|\theta^{(n)}\right\|_{F_{p, q}^{s}}\left\|\theta^{(n+1)}\right\|_{F_{p, q}^{s}} d \tau,
\end{aligned}
$$


where we used the fact that $\left\|S_{n+2} \theta_{0}\right\| \leq\left\|\theta_{0}\right\|$, Sobolev embedding theorem $F_{p, q}^{s-1} \hookrightarrow$ $L^{\infty}$ for $s-1>d / p$, (1.4) and the boundedness of the Calderón-Zygmund singular integral operator on $F_{p, q}^{s}$. Equation (3.15) together with the Gronwall inequality implies that

$$
\left\|\theta^{(n+1)}(t)\right\|_{F_{p, q}^{s}} \leq\left\|\theta_{0}\right\|_{F_{p, q}^{s}} \exp \left(C \int_{0}^{t}\left\|\theta^{(n)}\right\|_{F_{p, q}^{s}} d \tau\right)
$$

for some $C>0$ independent of $n$. Thus, if we choose $T_{0}=T_{0}\left(\left\|\theta_{0}\right\|_{F_{p, q}^{s}}\right)>0$ such that

$$
T_{0}\left\|\theta_{0}\right\|_{F_{p, q}^{s}} \leq \frac{1}{2 C} \ln 2
$$

we have, for any $n \in \mathbb{N}_{0}$,

$$
\sup _{0 \leq t \leq T_{0}}\left\|\theta^{(n+1)}(t)\right\|_{F_{p, q}^{s}} \leq 2 C\left\|\theta_{0}\right\|_{F_{p, q}^{s}}
$$

by the standard induction arguments. Then, $\theta^{(n+1)} \in C\left(\left[0, T_{0}\right] ; F_{p, q}^{s}\left(\mathbb{R}^{d}\right)\right)$. Moreover, it follows from Proposition 2.1 that

$$
\begin{aligned}
\left\|\partial_{t} \theta^{(n+1)}(t)\right\|_{F_{p, q}^{s-1}} & =\left\|u^{(n)} \cdot \nabla \theta^{(n+1)}\right\|_{F_{p, q}^{s-1}} \\
& \leq C\left(\left\|u^{(n)}\right\|_{L^{\infty}}\left\|\nabla \theta^{(n+1)}\right\|_{F_{p, q}^{s-1}}+\left\|u^{(n)}\right\|_{F_{p, q}^{s-1}}\left\|\nabla \theta^{(n+1)}\right\|_{L^{\infty}}\right) \\
& \leq C\left\|\theta^{(n)}\right\|_{F_{p, q}^{s-q}}\left\|\theta^{(n+1)}\right\|_{F_{p, q}^{s}}
\end{aligned}
$$

by Sobolev embedding and the boundedness of the Calderón-Zygmund singular integral operator on $F_{p, q}^{s}$, and then

$$
\sup _{0 \leq t \leq T_{0}}\left\|\partial_{t} \theta^{(n+1)}(t)\right\|_{F_{p, q}^{s}} \leq C\left\|\theta_{0}\right\|_{F_{p, q}^{s}}^{2}
$$

which implies that $\partial_{t} \theta^{(n+1)} \in C\left(\left[0, T_{0}\right] ; F_{p, q}^{s-1}\left(\mathbb{R}^{d}\right)\right)$. This together with (3.17) gives the uniform estimate of $\theta^{(n)}(x, t)$ in $n$.

Step 3. Existence.

We will show that there exists a positive time $T_{1}\left(\leq T_{0}\right)$ independent of $n$ such that $\theta^{(n)}$ and $u^{(n)}$ are Cauchy sequences in $X_{T}^{s-1} \triangleq \mathcal{C}\left(\left[0, T_{1}\right] ; F_{p, q}^{s-1}\right)$. For this purpose, we set

$$
\delta \theta^{(n+1)}=\theta^{(n+1)}-\theta^{(n)}, \quad \delta u^{(n+1)}=u^{(n+1)}-u^{(n)} .
$$

Then, it follows that $\delta \theta^{(n+1)}$ satisfies the equations

$$
\left\{\begin{array}{l}
\partial_{t} \delta \theta^{(n+1)}+u^{(n)} \cdot \nabla \delta \theta^{(n+1)}=-\delta u^{(n)} \cdot \nabla \theta^{(n)}, \\
\left.\delta \theta^{(n+1)}\right|_{t=0}=\Delta_{n+1} \theta_{0} .
\end{array}\right.
$$

Applying $\Delta_{k}$ to the first equation of (3.19), we get

$$
\partial_{t} \Delta_{k} \delta \theta^{(n+1)}+u^{(n)} \cdot \nabla \Delta_{k} \delta \theta^{(n+1)}=\left[u^{(n)}, \Delta_{k}\right] \cdot \nabla \delta \theta^{(n+1)}-\Delta_{k}\left(\delta u^{(n)} \cdot \nabla \theta^{(n)}\right) .
$$


Exactly as in the proof of (3.7), we get

$$
\begin{aligned}
& \left\|\delta \theta^{(n+1)}\right\|_{\dot{F}_{p, q}^{s-1}} \\
& \leq C\left\|\Delta_{n+1} \theta_{0}\right\|_{\dot{F}_{p, q}^{s-1}}+\int_{0}^{t}\|\| 2^{k(s-1)}\left(\left[u^{(n)}, \Delta_{k}\right] \cdot \nabla \delta \theta^{(n+1)}\right)(\tau)\left\|_{\ell^{q}(\mathbb{Z})}\right\|_{p} d \tau \\
& +\int_{0}^{t}\left\|\delta u^{(n)} \cdot \nabla \theta^{(n)}(\tau)\right\|_{\dot{F}_{p, q}^{s-1}} d \tau \\
& \leq C\left\|\Delta_{n+1} \theta_{0}\right\|_{\dot{F}_{p, q}^{s-1}}+\int_{0}^{t}\left(\left\|\nabla u^{(n)}\right\|_{L^{\infty}}\left\|\delta \theta^{(n+1)}\right\|_{\dot{F}_{p, q}^{s-1}}+\left\|\delta \theta^{(n+1)}\right\|_{L^{\infty}}\left\|\nabla u^{(n)}\right\|_{\dot{F}_{p, q}^{s-1}}\right) d \tau \\
& +\int_{0}^{t}\left(\left\|\delta u^{(n)}\right\|_{L^{\infty}}\left\|\nabla \theta^{(n)}\right\|_{\dot{F}_{p, q}^{s-1}}+\left\|\delta u^{(n)}\right\|_{\dot{F}_{p, q}^{s-1}}\left\|\nabla \theta^{(n)}\right\|_{L^{\infty}}\right) d \tau \\
& \leq C\left\|\Delta_{n+1} \theta_{0}\right\|_{\dot{F}_{p, q}^{s-1}}+\int_{0}^{t}\left(\left\|u^{(n)}\right\|_{F_{p, q}^{s}}\left\|\delta \theta^{(n+1)}\right\|_{F_{p, q}^{s}}+\left\|\delta u^{(n)}\right\|_{F_{p, q}^{s-1}}\left\|\theta^{(n)}\right\|_{F_{p, q}^{s}}\right) d \tau \\
& \leq C\left\|\Delta_{n+1} \theta_{0}\right\|_{\dot{F}_{p, q}^{s-1}}+\int_{0}^{t}\left(\left\|\theta^{(n)}\right\|_{F_{p, q}^{s}}\left\|\delta \theta^{(n+1)}\right\|_{F_{p, q}^{s}}+\left\|\delta \theta^{(n)}\right\|_{F_{p, q}^{s-1}}\left\|\theta^{(n)}\right\|_{F_{p, q}^{s}}\right) d \tau,
\end{aligned}
$$

where we used Proposition 2.1, Proposition 2.3, the embedding $F_{p, q}^{s-1} \hookrightarrow L^{\infty}$, and the boundedness of the Calderón-Zygmund singular integral operator on $F_{p, q}^{s}$. Thanks to the Fourier support of $\Delta_{n+1} \theta_{0}$, we have

$$
\left\|\Delta_{n+1} \theta_{0}\right\|_{\dot{F}_{p, q}^{s-1}} \leq C 2^{-(n+1)}\left\|\theta_{0}\right\|_{\dot{F}_{p, q}^{s}}
$$

Now, we estimate the $L^{p}$ norm of $\delta \theta^{(n+1)}$. Multiplying $\left|\delta \theta^{(n+1)}\right|^{p-2} \delta \theta^{(n+1)}$ on both sides of the first equation of (3.19), and integrating the resulting equations over $\mathbb{R}^{d}$, we obtain

$$
\begin{aligned}
\left\|\delta \theta^{(n+1)}(t)\right\|_{L^{p}} & \leq\left\|\Delta_{n+1} \theta_{0}\right\|_{L^{p}}+\int_{0}^{t}\left\|\delta u^{(n)} \cdot \nabla \theta^{(n)}(\tau)\right\|_{L^{p}} d \tau \\
& \leq 2^{-(n+1)}\left\|z_{0}^{+}\right\|_{\dot{F}_{p, q}^{s}}+C \int_{0}^{t}\left\|\delta u^{(n)}\right\|_{L^{p}}\left\|\nabla \theta^{(n)}\right\|_{L^{\infty}} d \tau \\
& \leq 2^{-(n+1)}\left\|z_{0}^{+}\right\|_{\dot{F}_{p, q}^{s}}+C \int_{0}^{t}\left\|\delta \theta^{(n)}\right\|_{L^{p}}\left\|\theta^{(n)}\right\|_{F_{p, q}^{s}} d \tau
\end{aligned}
$$

which together with (3.21) and (3.22) gives

$$
\begin{aligned}
\left\|\delta \theta^{(n+1)}\right\|_{F_{p, q}^{s-1} \leq} & C 2^{-(n+1)}\left\|\theta_{0}\right\|_{F_{p, q}^{s}}+C \int_{0}^{t}\left(\left\|\theta^{(n)}\right\|_{F_{p, q}^{s}}\left\|\delta \theta^{(n+1)}\right\|_{F_{p, q}^{s-1}}\right. \\
& \left.+\left\|\delta \theta^{(n)}\right\|_{F_{p, q}^{s-1}}\left\|\theta^{(n)}\right\|_{F_{p, q}^{s}}\right) d \tau \\
\leq & C 2^{-(n+1)}\left\|\theta_{0}\right\|_{F_{p, q}^{s}}+C T \sup _{t \in[0, T]}\left\|\theta^{(n)}\right\|_{F_{p, q}^{s} \|}\left\|\delta \theta^{(n+1)}\right\|_{F_{p, q}^{s-1}} \\
& +C T \sup _{t \in[0, T]}\left\|\theta^{(n)}\right\|_{F_{p, q}^{s}\left\|\delta \theta^{(n)}\right\|_{F_{p, q}^{s-1}}}
\end{aligned}
$$

Equation (3.23) together with (3.17) yields

$$
\begin{aligned}
\left\|\delta \theta^{(n+1)}\right\|_{X_{T}^{s-1}} \leq & C_{1} 2^{-(n+1)}+C_{1} T\left\|\delta \theta^{(n+1)}\right\|_{X_{T}^{s-1}} \\
& +C_{1} T\left\|\delta \theta^{(n)}\right\|_{X_{T}^{s-1}},
\end{aligned}
$$


where $C_{1}=C_{1}\left(\left\|\theta_{0}\right\|_{F_{p, q}^{s}}\right)$. Thus, if $C_{1} T \leq \frac{1}{4}$, then

$$
\left\|\delta \theta^{(n+1)}\right\|_{X_{T}^{s-1}} \leq C_{1} 2^{-n}+2 C_{1} T\left\|\delta \theta^{(n)}\right\|_{X_{T}^{s-1}} .
$$

This implies that

$$
\left\|\delta \theta^{(n+1)}\right\|_{X_{T}^{s-1}} \leq 2 C_{1} 2^{-(n+1)}
$$

Thus, $\left\{\theta^{(n)}\right\}_{n \in \mathbb{N}_{0}}$ is a Cauchy sequence in $X_{T_{1}}^{s-1}$. By the standard argument, for $T_{1} \leq$ $\min \left\{T_{0}, \frac{1}{4 C_{1}}\right\}$, the limit $\theta \in X_{T_{1}}^{s}$ solves (1.1) with the initial data $\theta_{0}$. The fact that $\theta \in$ $\operatorname{Lip}\left([0, T] ; F_{p, q}^{s}\right)$ follows from the uniform estimate (3.18).

Step 4. Uniqueness.

Consider $\tilde{\theta} \in C\left(\left[0, T_{1}\right] ;\left(F_{p, q}^{s}\right)\right)$ is another solution to (1.1) with the same initial data. Let $\delta \theta=\theta-\tilde{\theta}$ and $\delta u=u-\tilde{u}$. Then $\delta \theta$ satisfies the following equation:

$$
\left\{\begin{array}{l}
\partial_{t} \delta \theta+u \cdot \nabla \delta \theta=-\delta u \cdot \nabla \tilde{\theta} \\
\left.\delta \theta\right|_{t=0}=0 .
\end{array}\right.
$$

In the same way as the derivation in (3.24), we obtain

$$
\|\delta \theta\|_{X_{T}^{s-1}} \leq C_{2} T\|\delta \theta\|_{X_{T}^{s-1}}
$$

for sufficiently small $T$. This implies that $\delta \theta \equiv 0$, i.e., $\theta \equiv \tilde{\theta}$.

Blow-up criterion.

For the $a$ priori estimate (3.12), we only need to dominate $\|\nabla u\|_{L^{\infty}}$ and $\|\nabla \theta\|_{L^{\infty}}$. From Proposition 2.2 and the boundedness of the Calderón-Zygmund operator from $\dot{F}_{\infty, \infty}^{0}$ into itself, we have

$$
\begin{aligned}
\|\nabla u\|_{L^{\infty}} & \leq C\left(1+\|\nabla u\|_{\dot{F}_{\infty, \infty}^{0}}\left(\log ^{+}\|\nabla u\|_{F_{p, q}^{s-1}}+1\right)\right) \\
& \leq C\left(1+\|\theta\|_{\dot{F}_{\infty, \infty}^{1}}\left(\log ^{+}\|\theta\|_{F_{p, q}^{s}}+1\right)\right) .
\end{aligned}
$$

Similarly,

$$
\|\nabla \theta\|_{L^{\infty}} \leq C\left(1+\|\theta\|_{\dot{F}_{\infty, \infty}^{1}}\left(\log ^{+}\|\theta\|_{F_{p, q}^{s}}+1\right)\right)
$$

Thus, the a priori estimate (3.12) gives

$$
\|\theta\|_{F_{p, q}^{s}} \leq C\left\|\theta_{0}\right\|_{F_{p, q}^{s}} \exp \left(C \int_{0}^{t}\|\theta(\tau)\|_{\dot{F}_{\infty, \infty}^{1}}\left(\log ^{+}\|\theta(\tau)\|_{F_{p, q}^{s}}+1\right) d \tau\right) .
$$

By the Gronwall inequality

$$
\|\theta\|_{F_{p, q}^{s}} \leq C\left\|\theta_{0}\right\|_{F_{p, q}^{s}} \exp \left(C \exp \left(\int_{0}^{t}\|\theta(\tau)\|_{\dot{F}_{\infty, \infty}^{1}} d \tau\right)\right)
$$

Therefore, if $\lim \sup _{t \nearrow T^{*}}\|\theta(t)\|_{F_{p, q}^{s}}=\infty$, then $\int_{0}^{T^{*}}\|\theta(t)\|_{\dot{F}_{\infty, \infty}^{1}} d t=\infty$. 
On the other hand, it follows from the Sobolev embedding $F_{p, q}^{s} \hookrightarrow W^{1, \infty} \hookrightarrow \dot{F}_{\infty, \infty}^{1}$ for $s>d / p+1$ that

$$
\begin{aligned}
\int_{0}^{T^{*}}\|\theta(t)\|_{\dot{F}_{\infty, \infty}^{1}} d t & \leq T^{*} \sup _{0 \leq \tau \leq T^{*}}\|\theta(\tau)\|_{\dot{F}_{\infty, \infty}^{1}} \\
& \leq T^{*} \sup _{0 \leq \tau \leq T^{*}}\|\nabla \theta(\tau)\|_{L^{\infty}} \\
& \leq T^{*} \sup _{0 \leq \tau \leq T^{*}}\|\theta(\tau)\|_{F_{p, q}^{s}} .
\end{aligned}
$$

Then $\int_{0}^{T^{*}}\|\theta(t)\|_{\dot{F}_{\infty, \infty}^{1}} d t=\infty$ implies $\lim \sup _{t \nearrow T^{*}}\|\theta(t)\|_{F_{p, q}^{s}}=\infty$.

\section{Competing interests}

The authors declare that they have no competing interests.

\section{Authors' contributions}

All authors contributed equally to the writing of this paper. All authors read and approved the final manuscript.

\section{Author details}

${ }^{1}$ College of Electrical and Information Engineering, Hunan University, Changsha, 410000, P.R. China. ${ }^{2}$ School of Electrical and Automation Engineering, Hefei University of Technology, Anhui Pro., Hefei, 230009, P.R. China.

\section{Acknowledgements}

This work was supported by the National Natural Science Funds of China for Distinguished Young Scholar under Grant No. 50925727, The National Defense Advanced Research Project Grant Nos. C1120110004, 9140A27020211DZ5102, the Key Grant Project of Chinese Ministry of Education under Grant No. 313018, and the Fundamental Research Funds for the Central Universities (2012HGCX0003)

\section{Received: 22 January 2014 Accepted: 24 March 2014 Published: 06 May 2014}

\section{References}

1. Castro, A, Córdoba, D, Gancedo, F, Orive, R: Incompressible flow in porous media with fractional diffusion. Nonlinearity 22,1791-1815 (2009)

2. Córdoba, D, Gancedo, F, Orive, R: Analytical behaviour of the two-dimensional incompressible flow in porous media. J. Math. Phys. 48, 1-19 (2007)

3. Abidi, H, Hmidi, T: On the global well posedness of the critical quasi-geostrophic equation. SIAM J. Math. Anal. 40 167-185 (2008)

4. Caffarelli, L, Vasseur, V: Drift diffusion equations with fractional diffusion and the quasi-geostrophic equations. Ann. Math. 171, 1903-1930 (2010)

5. Chen, Q, Miao, C, Zhang, Z: A new Bernstein's inequality and the 2D dissipative quasigeostrophic equation. Commun. Math. Phys. 271, 821-838 (2007)

6. Córdoba, A, Córdoba, D: A maximum principle applied to the quasi-geostrophic equations. Commun. Math. Phys. 249, 511-528 (2004)

7. Kislev, A, Nazarov, F, Volberg, A: Global well-posedness for the critical 2D dissipative quasi-geostrophic equation. Invent. Math. 167, 445-453 (2007)

8. Wu, J: Global solutions of the 2D dissipative quasi-geostrophic equation in Besov spaces. SIAM J. Math. Anal. 36 1014-1030 (2004)

9. Córdoba, D, Faraco, D, Gancedo, F: Lack of uniqueness for weak solutions of the incompressible porous media equation. Arch. Ration. Mech. Anal. 200, $725-746$ (2011). doi:10.1007/s00205-010-0365-z

10. Chae, D: On the well-posedness of the Euler equations in the Triebel-Lizorkin spaces. Commun. Pure Appl. Math. 55 654-678 (2002)

11. Chae, D: On the Euler equations in the critical Triebel-Lizorkin spaces. Arch. Ration. Mech. Anal. 170, 185-210 (2003)

12. Chae, D: The quasi-geostrophic equations in the Triebel-Lizorkin spaces. Nonlinearity 16, 479-495 (2003)

13. Chen, Q, Miao, C, Zhang, Z: On the well-posedness of the ideal MHD equations in the Triebel-Lizorkin spaces. Arch. Ration. Mech. Anal. 195, 561-578 (2010). doi:10.1007/s00205-008-0213-6

14. Triebel, H: Theory of Function Spaces. Monograph in Mathematics, vol. 78. Birkhäuser Verlag, Basel (1983)

15. Chemin, J-Y: Perfect Incompressible Fluids. Oxford University Press, New York (1998)

16. Duoandikoetxea, J: Fourier Analysis. GSM, vol. 29. Am. Math. Soc., Providence (2001). (Translated and revised by D. Cruz-Uribe) 